AIAA-98-0291

\title{
IMAGING FOURIER TRANSFORM SPECTROMETERS FOR ENVIRONMENTAL SENSING
}

\author{
Leonard John Otten III, Associate Fellow, \\ Vice President. \\ Andrew D. Meigs, \\ Senior Scientist. \\ Bernard A. Jones, \\ Senior Designer. \\ Kestrel Corporation, Albuquerque, NM 87109 \\ R. Glenn Sellar \\ Optical Designer, \\ CREOL, Orlando, FL \\ J. Bruce Rafert \\ Chairman, Physics Department, \\ Michigan Technological University, Houghton, MI
}

\begin{abstract}
$\underline{\text { Abstract }}$
Spectral imaging has become a powerful new tool in environmental remote sensing from both air and space based platforms with a variety of sensor designs now in routine operation. One of the sensing technologies that has an ability to collect spectral data over a wide band is the spatially modulated Fourier transform hyperspectral imager (FTHSI). These sensors have been shown to operate from the near ultraviolet to the long wave infrared and are particularly well suited for use in the longer wavelengths and for observations from moving platforms. Recent FTHSI research has discovered techniques capable of producing wavenumber spectral resolution and small, affordable, imagers with real time data analysis capabilities.
\end{abstract}

\section{$\underline{\text { Introduction }}$}

The advantages of hyperspectral imaging in environmental mapping are now being recognized as the next step in optical remote sensing. Existing airborne observation systems using hyperspectral imaging have been assembled for remote sensing applications ${ }^{1}$. In addition, several hyperspectral imaging sensors have been designed for space flight, the most notable being the NASA sponsored Lewis sensor built by TRW, the MSTI 3 sensor, and the MightySat II.1 sensor being built for the Air Force Research Laboratory. To date, the majority of the aircraft instruments and most of the spacecraft designs have been based on diffraction technology using either a grating or dispersive element and usually some type of scanning mechanism. A spatially modulated Fourier transform based instrument is an alternative to the scanned dispersive system.

Several features inherent in a spatially modulated Fourier transform imager make it an attractive technology for environmental remote sensing:

- The very high optical throughput inherent in a Fourier based instrument means that sufficient photons will be available.

- The unique capability of a Fourier transform imager to decouple spectral and spatial resolution. No other spectral sensing technologies have this feature. All dispersive configurations have their spectral resolution directly tied to their spatial resolution. In a dispersive design, an improvement in spectral resolution requires a narrowing of the entrance slit which in turn decreases the optical throughput and the spatial resolution causing dramatic losses in signal to noise and signal to 
clutter ratios. In a Fourier design the spectral resolution is completely independent of the sensor foreoptics or entrance field stop.

- A Spatially modulated FTHSI can simultaneously record a complete spectrum: spectral co-registration is assured. No scanning mechanisms are required, allowing these sensors to be used in temporally varying applications while adding a high degree of ruggedness.

- Using an intra-interferometer dispersive technique, the spectral range can be tailored to a definable band limited only by the detector sensitivity allowing Fourier transform sensors a great deal of latitude in spectral band width selection. With this technique the Nyquist limit can be applied only to the bandwidth of interest thus increasing the spectral resolution significantly.

- A FTHSI design has the Connes advantage where the wavenumber scale and instrument line shape are precisely determined and independent of wavenumber. This feature allows precise, unambiguous, absolute spectral calibration.

These advantages make the FTHSI uniquely suited to current remote sensing interest in an ultraspectral sensor and in seeking means to reduce the cost and amounts of data that must be handled by a hypersepctral sensor.

\section{Ultra Spectral Imaging Spectrometers}

A recent focus of the remote sensing community has been in finding methods that produce finer spectral resolution'. Current hyperspectral imagers typically have hundreds (50 to 200 bands) of 10 to $50 \mathrm{~nm}$ spectral bands. A new generation of hyperspectral instruments called ultraspectral imagers (USI) is now being developed to satisfy a growing interest in increased spectral resolution. These devices have sufficient spectral resolution to allow molecular absorption or emission bands to be imaged in two dimensions from moving platforms.

As an example of what a USI can resolve, Figure 1 illustrates the spectral resolution required to identify specific lines of a typical hydrocarbon gas, benzene. The limitations of current instruments represented by a grating type hyperspectral sensor (HSI) ${ }^{2}$, and a $100 \mathrm{~cm}^{-1}$ hyperspectral device such as Kestrel's FTHSI are noted ${ }^{3}$. As this figure illustrates, a sensor with better than $2 \mathrm{~cm}^{-1}$ resolution is required to positively identify this specific molecule. Coarser spectral resolutions are not capable of unambiguous typing.

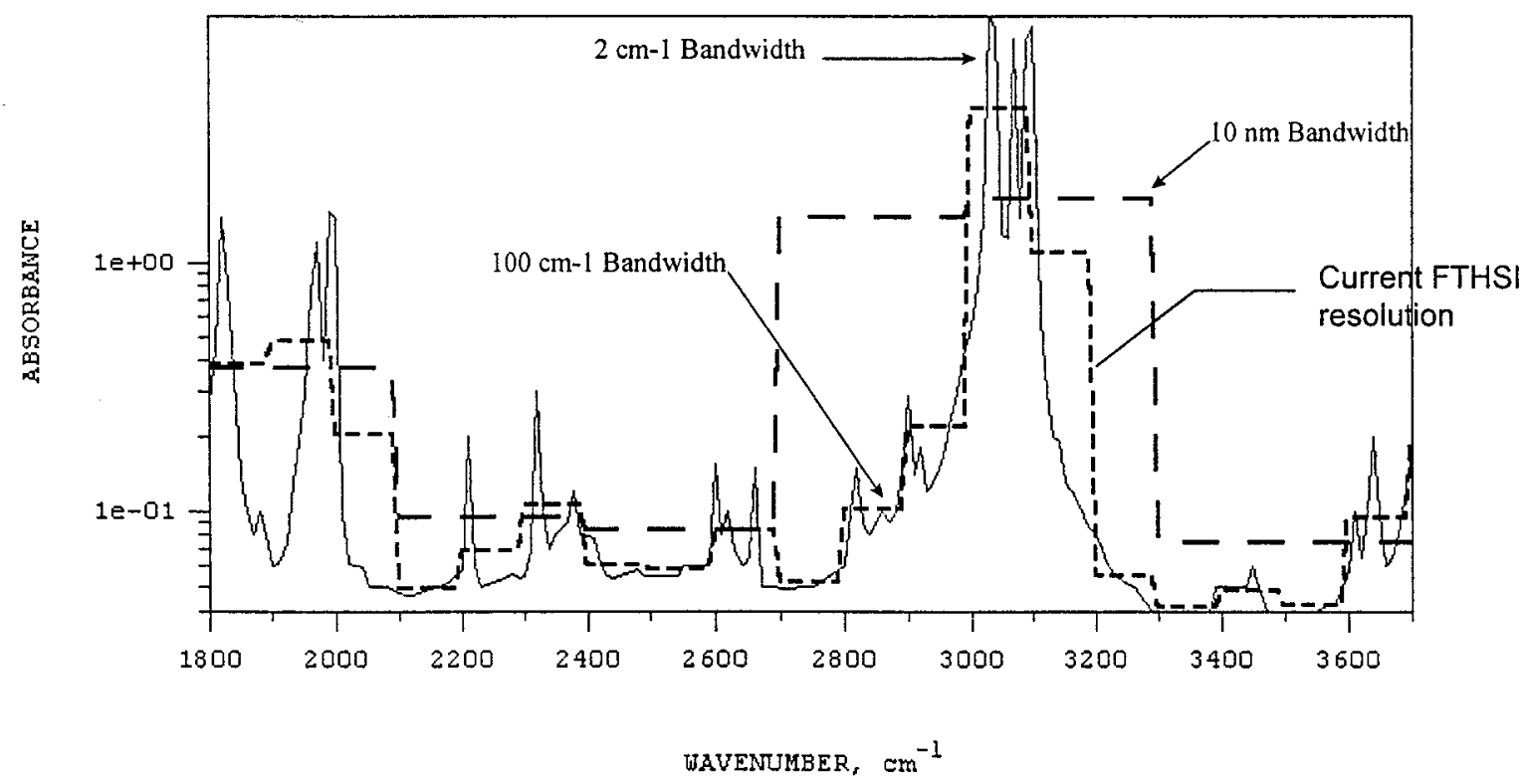

Figure 1. An ultraspectral imager resolution of $<2 \mathrm{~cm}^{-1}$ permits identification of individual molecular species, a feature missing from all available alternative imaging systems. Conventional grating with $10 \mathrm{~nm}$ resolution, and FTHSI instruments with $100 \mathrm{~cm}^{-1}$ resolution, are too coarse to reveal the distinct molecular absorption lines required for positive identification. 
A sensor with these capabilities can uniquely identify and map materials by their molecular absorption or emission signatures. Discrimination against backgrounds increase rapidly as the spectral bandwidth is decreased to the width of molecular lines. The reasons for these improvements are two fold. First, with molecular feature resolution, an ultraspectral imager can isolate a specific material's signature, replacing cross correlation search techniques with a simple fitting algorithm ${ }^{4}$. Second, for highly mixed background, and targets that are spatially smaller than a pixel, more and better defined end members can be determined to improve sub-pixel unmixing ${ }^{s}$.

To have wide use, a commercial USI sensor needs to have both MWIR and LWIR coverage, provide several hundreds of spectral bands, have a spatial resolution that allows both reasonable search capabilities and sufficient spatial resolution for target or feature recognition. In specific quantities, these performance requirements are:
Spectral bands: $3000 \mathrm{~m}$ to $5000 \mathrm{~nm}$ $8000 \mathrm{~nm}$ to $12000 \mathrm{~nm}$

Spatial resolution: $<2 \mathrm{mrad}$

Field of view: $>0.23 \mathrm{rad}$

FWHM spectral resolution: $<2 \mathrm{~cm}^{-1}$

To our knowledge no available sensor achieves all these requirements, Table 1 . The sensor discussed in this paper will be the first.

A Fourier transform based imager using spatial modulation appears to be one of the few technologies that can be extended to produce an ultraspectral imager, especially in the IR bands. By taking advantage of the FTHSI features noted above it is possible to design a medium wave infrared (MWIR) and long wave infrared (LWIR) ultra high resolution passive spectral imager that can operate from a moving platform or observe a time changing scene. Spatially modulated Fourier transform instruments have been assembled previously for

Table 1

General Specification of Available High Spectral Resolution Imagers

\begin{tabular}{|c|c|c|c|c|c|c|}
\hline $\begin{array}{l}\text { System, Name } \\
\text { and Source }\end{array}$ & $\begin{array}{l}\text { AVIRIS } \\
\text { NASA JPL }\end{array}$ & $\begin{array}{l}\text { CASI } \\
\text { Itri Corp }\end{array}$ & $\begin{array}{l}\text { TRWIS } \\
\text { II/III } \\
\text { TRW }\end{array}$ & $\begin{array}{l}\text { FTHSI } \\
\text { Kestrel }\end{array}$ & $\begin{array}{l}\text { HYDICE } \\
\text { ERIM }\end{array}$ & $\begin{array}{l}\text { MWIR/ LWIR } \\
\text { dual band USI } \\
\text { Kestrel }\end{array}$ \\
\hline $\begin{array}{l}\text { Basic design } \\
\text { concept }\end{array}$ & $\begin{array}{l}\text { 1D Array } \\
\text { whisk- } \\
\text { broom } \\
\text { grating }\end{array}$ & $\begin{array}{l}\text { D array } \\
\text { pushbroom } \\
\text { grating }\end{array}$ & $\begin{array}{l}\text { D array } \\
\text { pushbroom } \\
\text { grating }\end{array}$ & $\begin{array}{l}\text { 2D array } \\
\text { pushbroom } \\
\text { Fourier }\end{array}$ & $\begin{array}{l}\text { pushbroom, } \\
\text { dispersive } \\
\text { wedge }\end{array}$ & $\begin{array}{l}\text { 2D Array } \\
\text { Pushbroom } \\
\text { dual band, } \\
\text { Fourier }\end{array}$ \\
\hline $\begin{array}{l}\text { Spectral range } \\
(\mathrm{nm})\end{array}$ & $410-2450$ & $423-946$ & $500-2300$ & $500-1050$ & $450-2500$ & $\begin{array}{l}3000-5000 \\
8000-12000\end{array}$ \\
\hline $\begin{array}{l}\text { \# of } \\
\text { simultaneous } \\
\text { spectral bands }\end{array}$ & 224 & 18 & 384 & 256 & 210 & 380 \\
\hline $\begin{array}{l}\text { Max spectral } \\
\text { resolution }\end{array}$ & $\begin{array}{l}9.6 \mathrm{~nm} \text { in } \\
\text { VIS-NIR } \\
20 \mathrm{~nm} \text { in IR }\end{array}$ & $10 \mathrm{~nm}$ & $\begin{array}{l}5.25 \mathrm{~nm} \text { in } \\
\text { VIS-NIR } \\
6.25 \mathrm{~nm} \text { in } \\
\text { IR }\end{array}$ & $87 \mathrm{~cm}^{-1}$ & $\begin{array}{l}8 \mathrm{~nm} \text { in } \\
\text { VIS-NIR } \\
20 \mathrm{~nm} \text { in IR }\end{array}$ & $\begin{array}{l}0.4 \mathrm{~cm}^{-1} \text { in } \\
\text { LWIR } \\
2.5 \mathrm{~cm}^{-1} \text { in } \\
\text { MWIR }\end{array}$ \\
\hline $\begin{array}{l}\text { Spatial } \\
\text { resolution } \\
\text { (mrad)/FOV } \\
(\mathrm{rad}) \\
\end{array}$ & $1.0 / 0.53$ & $2.4 / 0.61$ & $0.9 / 0.23$ & $0.6 / 0.26$ & $0.5 / 0.23$ & $2.0 / 0.22$ \\
\hline $\begin{array}{l}\text { \# of arrays and } \\
\text { band }\end{array}$ & $\begin{array}{l}\text { three (VIS- } \\
\text { NIR, IR, and } \\
\text { MWIR) }\end{array}$ & $\begin{array}{l}\text { one } \\
\text { (VIS-NIR) }\end{array}$ & $\begin{array}{l}\text { two } \\
\text { (VIS, IR) }\end{array}$ & $\begin{array}{l}\text { one } \\
\text { (VIS-NIR) }\end{array}$ & $\begin{array}{l}\text { one } \\
\text { multilayer } \\
\text { (VIS to IR) }\end{array}$ & $\begin{array}{l}\text { two } \\
\text { (MWIR } \\
\text { LWIR) }\end{array}$ \\
\hline Estimated cost & $\$ 50 \mathrm{M}+$ & $\$ 0.40 \mathrm{M}+$ & $\$ 1 \mathrm{M}+$ & $\$ 0.250 \mathrm{M}$ & $\$ 50 \mathrm{M}+$ & $\$ 0.400 \mathrm{M}$ \\
\hline
\end{tabular}


ground based imaging applications $6,7,8$ and several sensors are now operating from airborne platforms 9,10 . These devices have in common the use of an interferometer to create an interferogram that contains the desired spectral information.

A sketch of the basic optical components is shown in Figure 2, a ray trace created using the ZEMAX optical design code of an instrument Kestrel has manufactured. The scene is observed through a set of foreoptics that images it onto a field stop, where the width of the stop is used only to set the along-track spatial resolution, not to determine the spectral resolution, a unique feature of these devices. In normal operation, the one dimensional image is then passed through the interferometer, shown as three elements, where the rays are split, slightly sheared, and recombined to create an interference pattern in one dimension. From the interferometer, a Fourier lens collimates the light and a cylindrical lens images the energy onto the detector, at the far right in the Figure, preserving the one by $\mathbf{n}$ spatial dimension and the interference pattern.

Two new techniques are used to create a FTUSI that operates into the MWIR and LWIR. The first changes the basic three element interferometer by incorporating a dispersive element and the second introduces a beam reshaping element between the Fourier lens and the detector to increase the number of spectral sample elements and offer an electronically selectable lower data rate search mode coupled to a high data rate, high resolution identification mode.

The dispersive element, originally investigated by Okamota, et.al. ${ }^{11}$, allows the designer to tailor the bins in which the shortest and longest wavelengths reside allowing us to apply the Nyquist criteria to only the band of interest. This technique produces a significant improvement over a conventional FTS that must apply the Nyquist criteria from the blue cut off to zero wavenumber. As shown in Figure 3 the resulting loss in spectral resolution in a conventional FTS in the MWIR and LWIR is about $50 \%$.

A sample of the increase in spectral resolution that can be obtained with the dispersive element is shown in Figures 4 and 5. The array size was assumed to be 512 detectors. The minimum and maximum wavenumber were assumed to be 800 $\mathrm{cm}^{-1}(12 \mu \mathrm{m})$ and $1200 \mathrm{~cm}^{-1}(8 \mu \mathrm{m})$, for Figure 4 and $2000 \mathrm{~cm}^{-1}(5 \mu \mathrm{m})$ and $3300 \mathrm{~cm}^{-1}(3 \mu \mathrm{m})$, Figure 5, respectively.

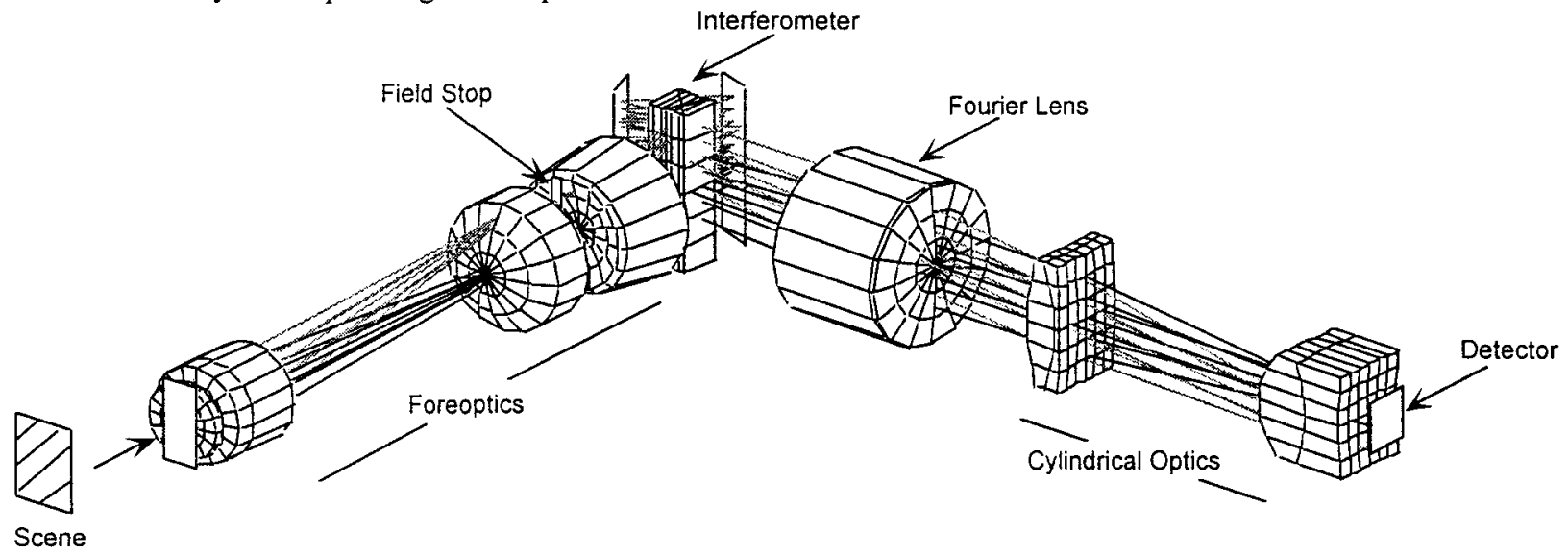

Figure 2. Basic components of a spatially modulated Fourier transform optical components. 


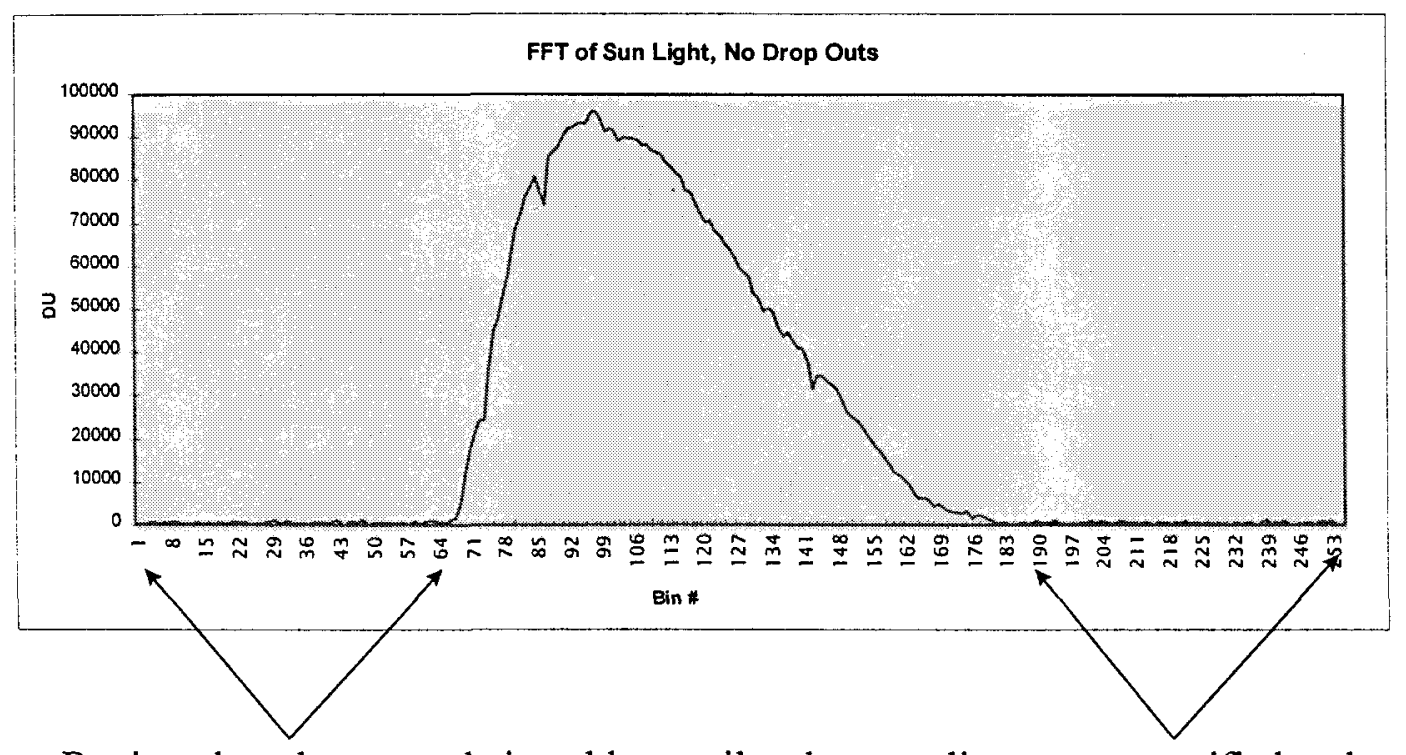

Regions lost due to not being able to tailor the sampling over a specific band.

Figure 3. Example of lost resolution in a conventional FTHSI. By using the tailoring technique proposed in this work, all of the sensor's pixels can be used across the bandwidth of interest.

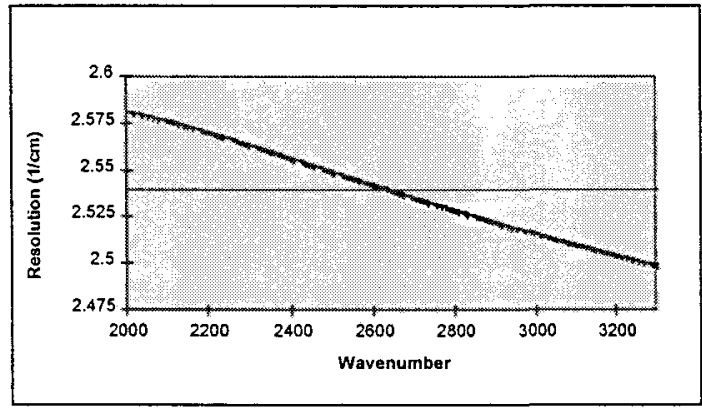

Figure 4 , The spectral resolution as a function of the wavenumber for a dispersive element intrainterferometer MWIR design.

All of the designs have a better spectral resolution than in a system without any dispersion, $\Delta \sigma=6.4 \mathrm{~cm}^{-1}$ for the MWIR case and $2.4 \mathrm{~cm}^{-1}$ for the LWIR sample. There is a slight variation in the resolution; for the longer wavelengths (smaller wavenumber) the enhancement is greater than for shorter wavelengths. The second technique employed is optical folding. A mirror arrangement was found that can be placed between the original image plane and the reimaging lens set to trade spatial resolution for spectral resolution. The major difficulty encountered in the design is that vignetting and crosstalk will start to occur if the optics are any faster than about $f / 8$. The design also has to maintain equal paths to preserve the interference pattern.

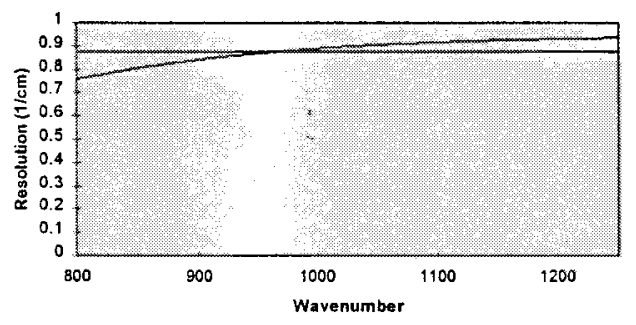

Figure 5, The spectral resolution as a function of the wavenumber for a dispersive element intrainterferometer LWIR design.

A three dimensional orthographic sketch of a mirror system that will take a $1536 \times 170$ array and re-stack it into a $512 \times 512$ array while maintaining equal paths is shown in Figure 6. This allows us to increase the sample size from 512 to 1536 for a three fold increase in spectral resolution.

When combined, these two techniques can be packaged into a compact unit easily mounted into an airframe. An example of a conceptual mechanical configurations for a MWIR and LWIR sensor is shown in Figure 7. 


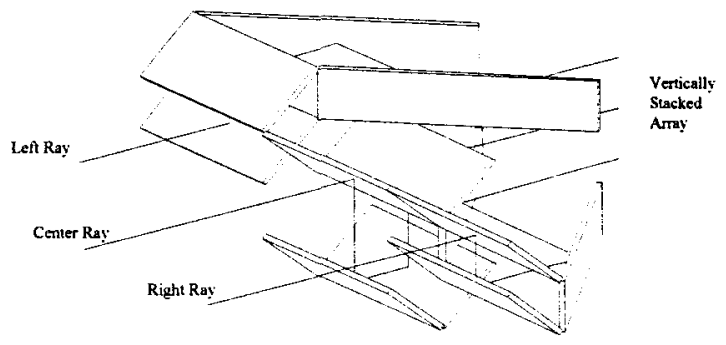

Figure 6 . Three dimensional view of the stacking mirror arrangement.

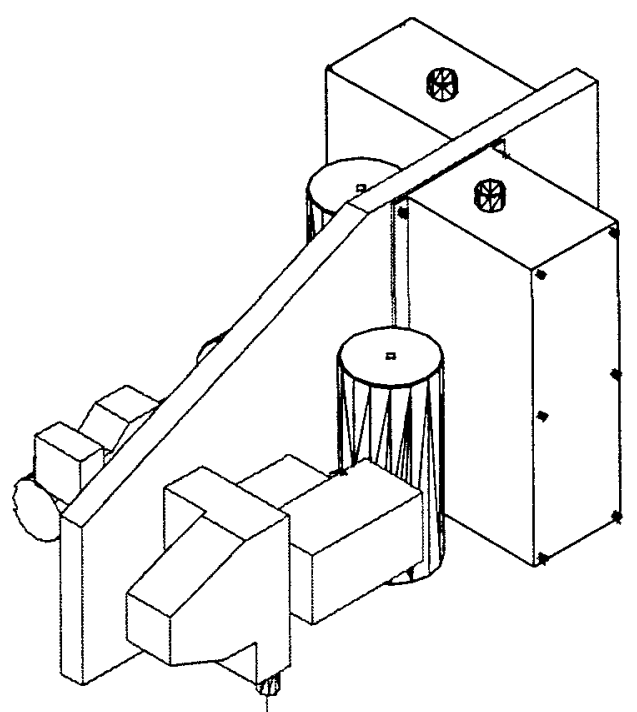

Figure 7. Overall configuration of a USI.

\section{Low Cost Hyper Spectral Sensors}

While the potential of hyperspectral imaging is now well recognized, the realization of this potential has been stymied by several factors. First, the availability of robust sensors that are easily fielded has limited the applications to a modest number of well funded, Government backed programs. Today no hyperspectral imager costing anywhere close to $\$ 100,000$ exists, making the costs of even collecting the data a high value task. Second, the volume of data collected and existing techniques for reducing and calibrating the information have caused the turn around time on data processing to be of such a length to make them useful in only the most relaxed applications. These limitations have renewed an interest in sensors that are available at reasonable costs and in finding a capability to replace the normal large volumes of data with a smaller set of information.
Fourier transform hyper-spectral imaging (FTHSI), has attributes that make it particularly attractive for solving these applications $12,13,14$. With savings over existing hyperspectral imagers such as NASA's Airborne Visible Infrared Imaging Spectrometer (AVIRIS), ERIM's HYDICE, TRW's TRWIS III, or Itre's Compact Airborne Spectrographic Imager (casi) ${ }^{15,16,17}$.The FTHSI technologies that have been fielded have shown that the underlying science is well founded and that the sensors have utility features that make them very attractive in both Government and commercial use. The development process has shown that the expected spatial and spectral resolution can be achieved and that the common path interferometer is quite robust. This research has also suggested manufacturing of a FTHSI based sensor for significantly less money than is normally associated with a sensor of these capabilities might be feasible.

In parallel with the advances made in FTHSI technology, Sandia National Laboratories has been conducting research in Real Time Signal and Image Processing (RTISP). As part of this development Sandia has developed high speed data reduction algorithms that offer real time assembly of RTSIP imagery using modern, COTS, PC processing. The basic Fourier transform algorithms employed in the RTSIP data analysis are the same as those required to reduce the FTHSI data. Applying these algorithms to an FTHSI sensor creates the same real time data presentation capability that is available in RTSIP. This advance represents a significant increase in the utility of FTHSI sensors by overcoming one of the long standing drawbacks in the employment of the technology, the lengthy data analysis time while simultaneously addressing the costly high speed and large volume data storage issue.

To have wide use as an environmental sensor, a low cost instrument must have both visible to near infrared and infrared coverage, provide several hundreds of spectral bands and have a spatial resolution that allows both reasonable search capabilities and sufficient spatial resolution for target or feature recognition. In specific quantities, these performance requirements are:

- $<100 \mathrm{~cm}-1$ spectral resolution 
- $\quad>180$ bands total (128 VIS-NIR, 54 MWIR)

- $<1 \mathrm{mrad}$ IFOV

- $>0.22 \mathrm{rad}$ FOV

- $0.5 \mu \mathrm{m}$ to $1.0 \mu \mathrm{m}$ and $1.7 \mu \mathrm{m}$ to $3 \mu \mathrm{m}$ bands collected simultaneously

- AC compatibility

- Internal frequency calibration

- Self contained except for power

- Incorporate a reflectance reference (downwelling)

- LOS with $>2$ mrad error when DGPS or PCode available

- Meet cost goal of less than $\$ 100,000$

- Meet real time data processing throughput at $15 \mathrm{fps}$ in the dual band mode

A parametric study was done to optimize a sensor that meets the above requirements list. Our cost analysis has shown that the choice of cameras is the dominant cost driver.

Because the detector dominates system cost, parameters that define its selection need to be carefully considered. For example, a larger dynamic range improves the performance of the system as seen in Figure 8, but for a significant cost increase ( $\$ 2,000$ vs. $\$ 20,000)$. A similar set of tradeoffs can be made in detector quantum efficiency and sensor noise. Figure 8 also shows the effect of a different quantum efficiency for a fixed optical design. Both the magnitude and spectral distribution have a strong influence on performance. These factors also relate to cost with the better quantum efficiencies coming from the more expensive backside illuminated detectors. Noise is found to be an expensive performance parameter to improve. The effect of random noise in a camera / data system is seen in Figure 9. As the noise approaches 8 bits, the data becomes difficult to use. Noise below 4 bits produces acceptable results.

The conclusion of these trades is that a low cost dual band HSI can be made with performance close to the limits shown earlier. Possible optical configurations for such a sensor are shown in Figures 10 and 11. These instruments are easily packaged into an aircraft compatible unit as seen in Figures 12 and 13. The estimated performance for the set of sensors is summarized in Figures 14 and 15.

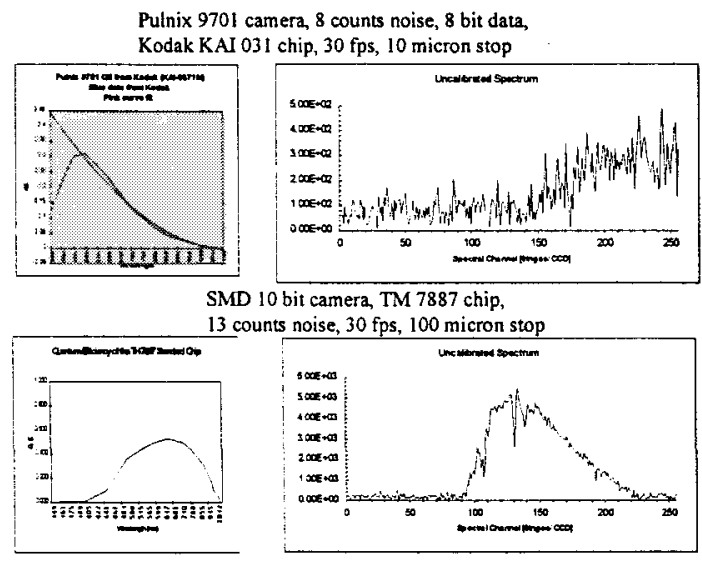

Figure 8. Detector quantum efficiency, seen on the left, strongly influence the performance of a FTHSI.
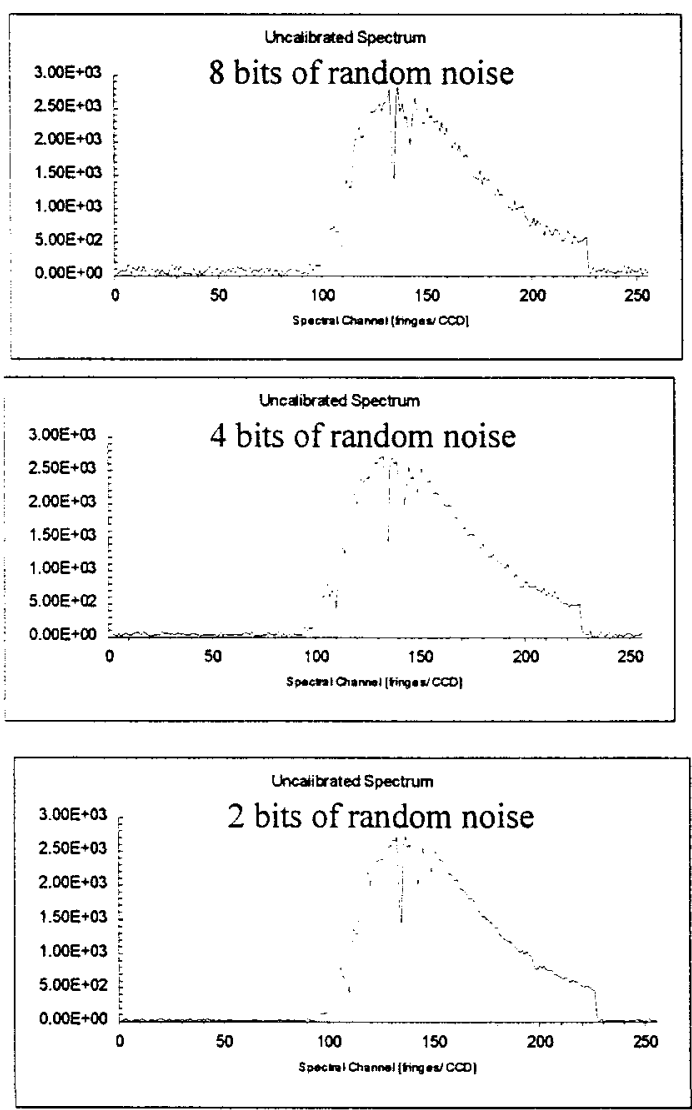

Figure 9. Effect of noise on a FTHSI. The optical system and quantum efficiency are the same for each example. Noise is noted by the ripple in the spectra. 


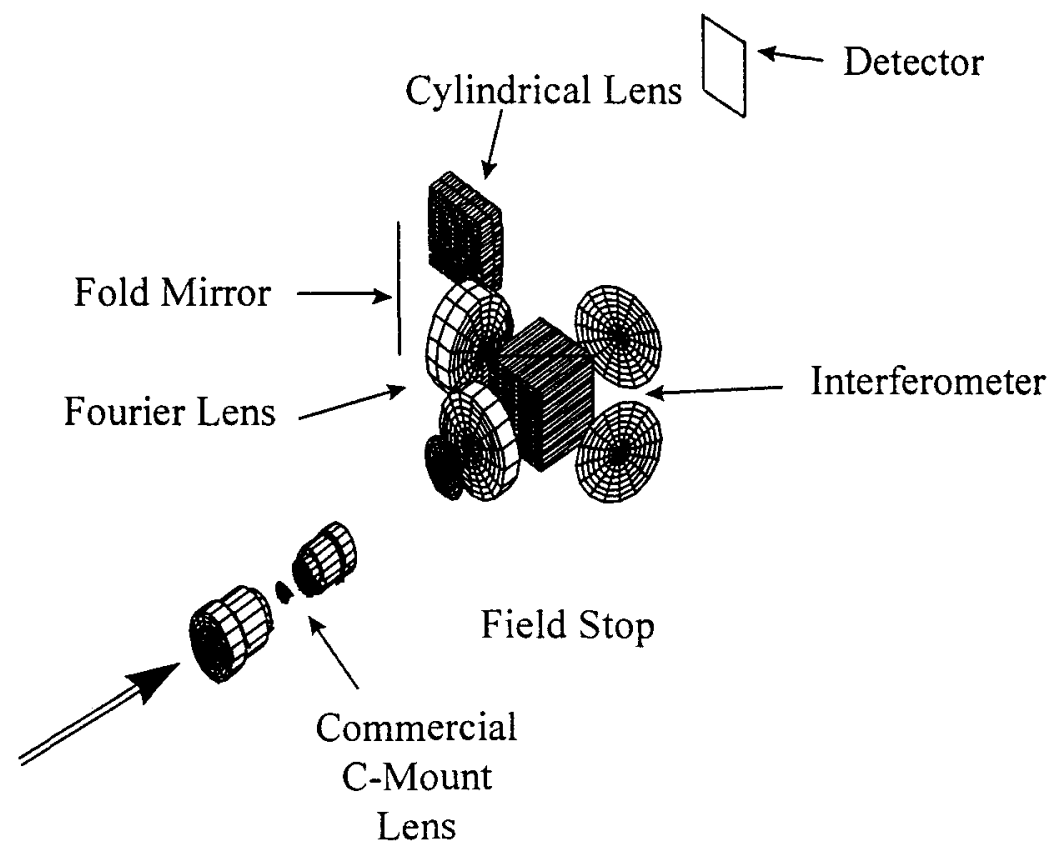

Figure 10. Optical layout for a low cost FTHSI. The overall size of this optical system allows it to fit within a coffee cup.

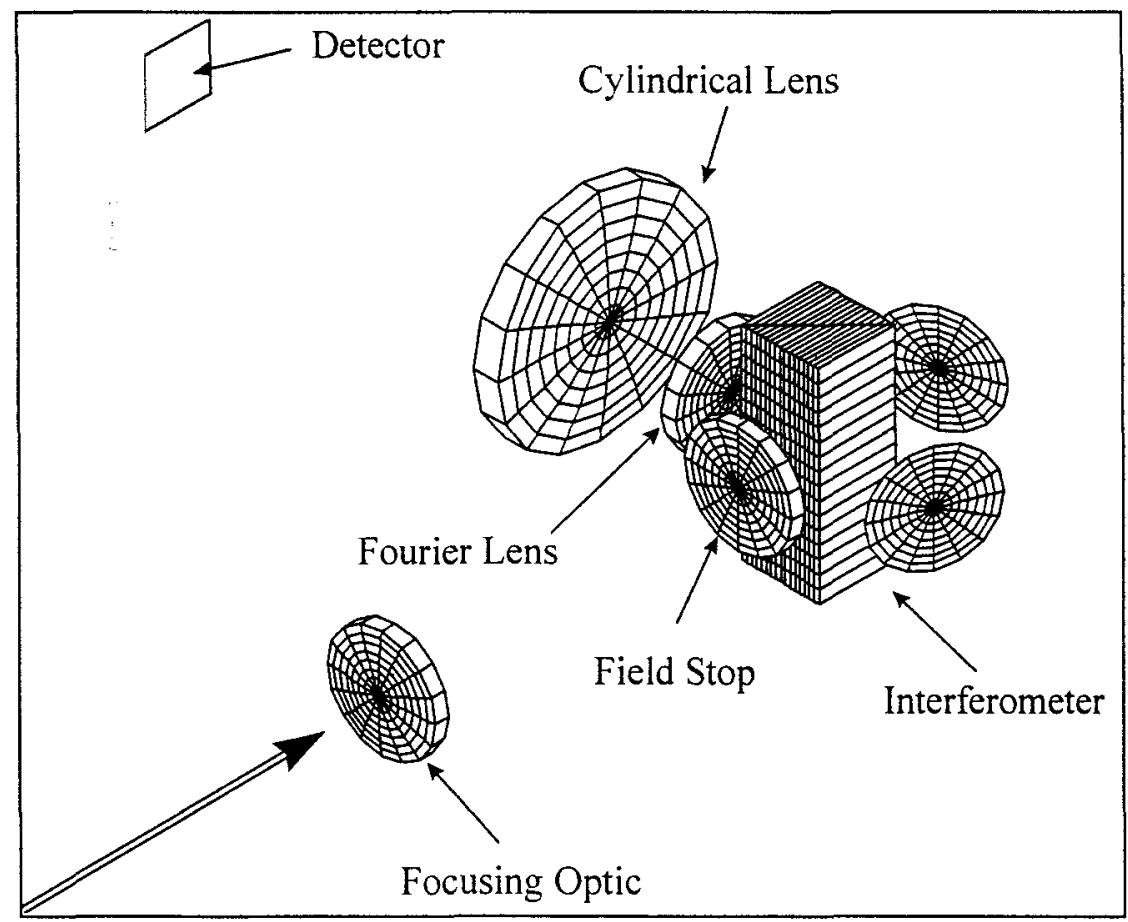

Figure 11. Optical layout for a low cost MWIR FTSHI. Again, the size is such that it can be easily held in one hand. 


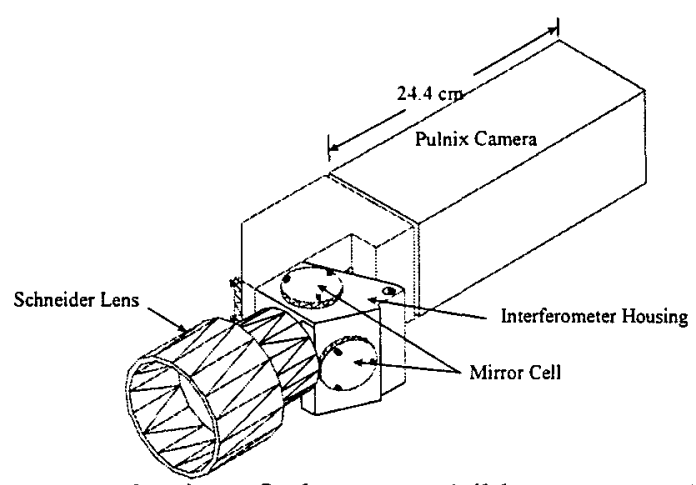

Figure 12. Packaging of a low cost visible to near IR FTHSI

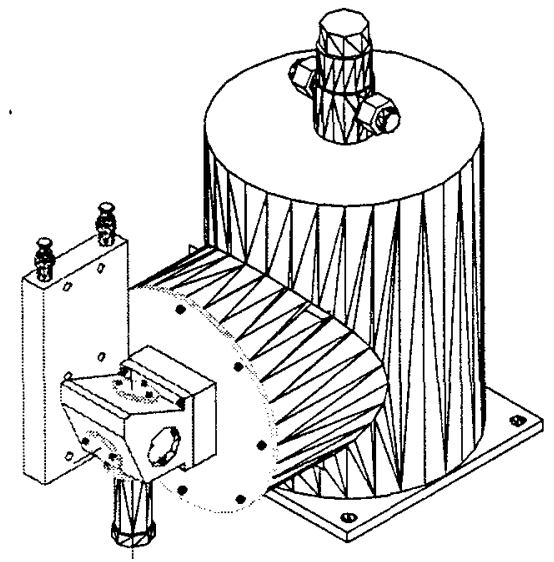

Figure 13 . Packaging of a low cost MWIR FTHSI

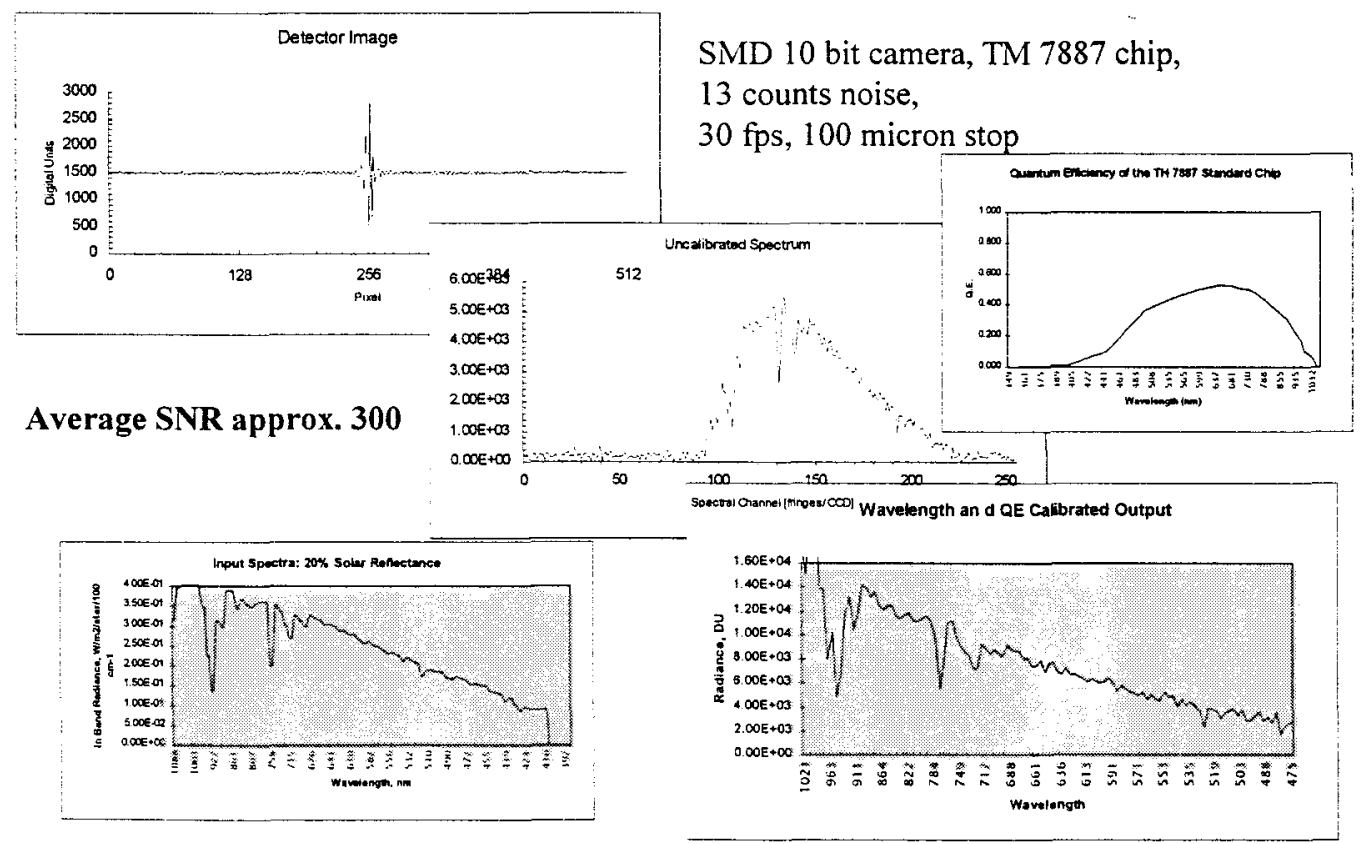

Figure 14. Estimated performance of a low cost visible to near IR HIS. 


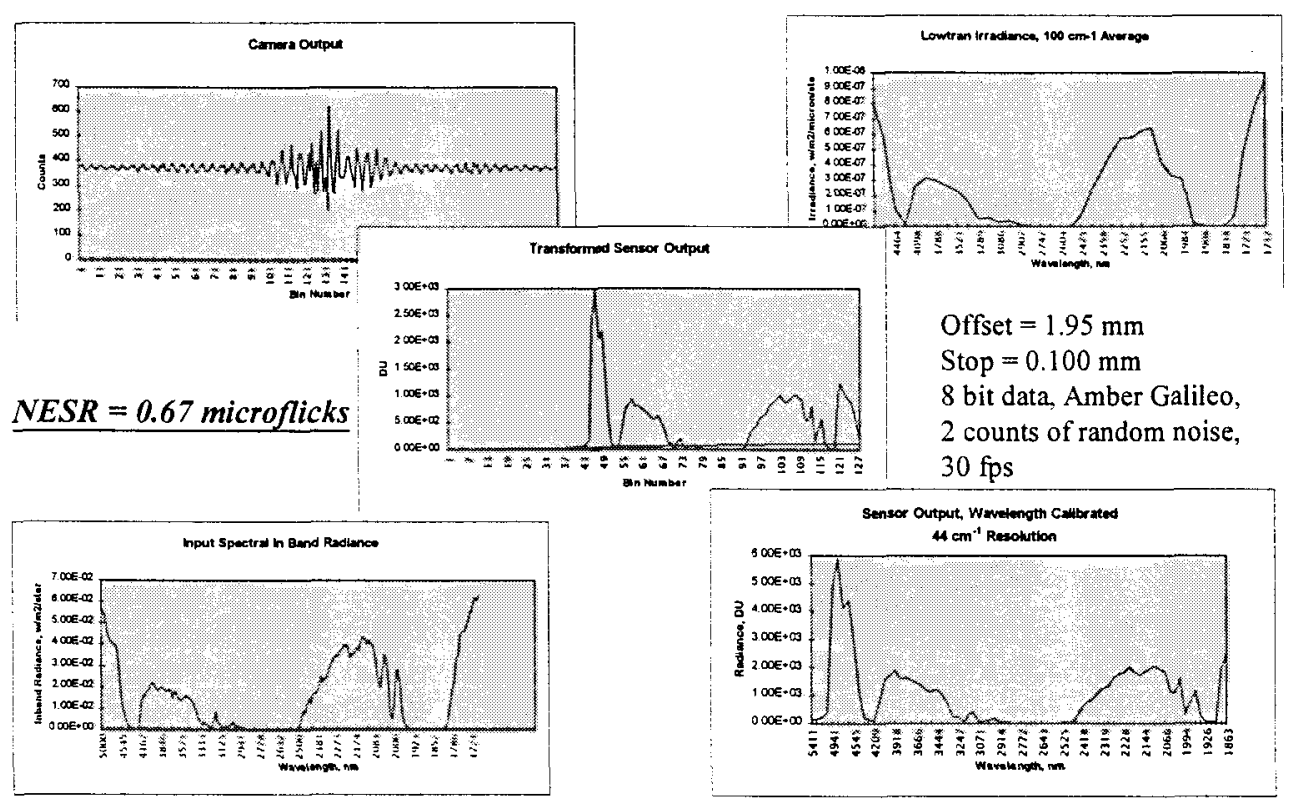

Figure 15. Estimated performance of a low cost MWIR HIS.

$\underline{\text { Summary }}$

Hyperspectral imaging is advancing in several new directions relevant to environmental remote sensing. New technologies based on Fourier transform spatially modulated interferometers are now making it possible to consider spectral imaging systems that have sufficient spectral resolution to map molecular absorption and emission lines. These ultraspectral sensors are opening new capabilities in mineral exploration, environmental monitoring, and chemical dispersion observations. The same basic technology is offering advances in the cost effectiveness of applying hyperspectral imaging by reducing the sensor cost and replacing conventional bulk raw data storage with information extracted by a real time processor.

\section{Acknowledgments}

This work was sponsored in part by the Department of Energy under Grant DE-FG0396ER86054/A002 and the Ballistic Missile Defense Organization under Contract F29601-C96-0033.

\section{$\underline{\text { References }}$}

${ }^{1}$ R. Anderson, W. Malila, R. Maxwell, and L. Reed, Military Utility of Multispectral and
Hyperspectral Sensors, ERIM, Ann Arbor, 2-2, 1994.

${ }^{2}$ M. A. Folkman, R. DeLong, C. Willoughby, D. Gleichauf, S. Thordarson, M. Figueora and W. Procino, "TRWIS III: An Aircraft-Based Hyperspectral Imager" Presented at IRIS Passive Sensors Conference 3/1996, Monterey, CA.

${ }^{3}$ L. J. Otten, A. D. Meigs, R. G. Sellar and J. B. Rafert, "Measured performance of an airborne Fourier transform hyperspectral imager", Imaging Spectrometry II, SPIE Proceedings 2819, 7-8 Aug, 1996, Denver, CO.

${ }^{4}$ Ibid. Military Utility of Multispectral and Hyperspectral Sensors, 6-29.

5 J. B. Adam and M. O. Smith, "Spectral mixture modeling: a new approach to analysis of rock and soil at the Viking lander 1 site," $J$. Geographic Research, V91, 8096-8112, 1986.

${ }^{6}$ J. B. Rafert, P. Lucey, and H. A. Newby, "A Spatially Modulated Imaging Fourier Transform Spectrometer for Astronomical and Booster Observations," Proceedings of the ESO Conference on the Progress in Telescope and Instrumentation Technology, ESO, Garching, 1992.

${ }^{7}$ J. B. Rafert, E. Holbert, H. Newby, S. Durham, E. Caudill and D. Keating, "The Malabar spatially modulated imaging Fourier transform spectrometer (SMIFTS)," Proceedings of the International Symposium on Spectral Sensing Research, Vol. 1, pp. 263-271, Nov., 1992. 
${ }^{8}$ L. J. Otten, R. G. Sellar, J. B. Rafert, and M. Gautreaux, "Hyperspectral measurements of common camouflages," Conference on Targets and Backgrounds: Characterization and Representation, Orlando, FL, SPIE 2469, April, 1995.

${ }^{9}$ P. G. Lucey, T. Williams, K. Horton, K. Hinck, C. Budney, J. B. Rafert and E. T. Rusk, "SMIFTS: A cryogenically cooled spatially modulated, imaging, Fourier transform spectrometer for remote sensing applications," Proceedings of the International Conference on Spectral Sensing Research, Vol. 1, pp. 251-262, Nov. 1992.

${ }^{10}$ P. D. Hammer and W. H. Smith, "Imaging interferometer for terrestrial remote sensing: digital array scanned interferometer (DASI) instrument development," Conference on Imaging Spectrometry, Orlando, FL, SPIE 2480, April 1995.

"Okmaoto, T, Kawata, S. and Minami, S, "Optical methods for resolution enhancement in photodiode array Fourier transform spectroscopy", ApOpt, 24:23, 4221-4225, 1 Dec 1985.

${ }^{12}$ L. J. Otten, J. B. Rafert, R. G. Sellar, "The design of an airborne Fourier transform visible hyperspectral imaging system for light aircraft environmental remote sensing", Conference on Imaging Spectrometry, Orlando FL, SPIE Conference Proceeding 2480, April, 1995.

${ }^{13}$ A. D. Meigs, L. J. Otten, E. W. Butler, B. A. Jones, F. P. Portigal, R. G. Sellar, "Kestrel's new FTVHSI instrument for hyperspectral remote sensing from light aircraft", Conference on Environmental Applications of Hyperspectral Remote Sensing, Taormina, Italy, SPIE Conference Proceeding 2960, 23-26 September, 1996.

${ }^{14}$ L. J. Otten, A. D. Meigs, R. Glenn Sellar, "Calibration and performance of the airborne Fourier Transform visible hyperspectral imager (FTVHSI)", Vol. I, 145-154, Second

International Airborne Remote Sensing Conference, San Francisco, CA, 24-27 June 1996.

${ }^{15}$ G. Borstad, "Ecosystem surveillance and monitoring with a portable airborne imaging spectrometer system", 1st Thematic Conference on Remote Sensing for Marine and Coastal Environments, New Orleans, LA, 15 June, 1992.

${ }^{16}$ R. K. De Long, T. E. Romesser, J. Marmo and M. A. Folkman, "Airborne and satellite imaging spectrometer development at TRW", Conference on Imaging Spectrometry, Orlando, FL, SPIE Conference Proceedings 2480, April 1995.

${ }^{17}$ R.W. Basedow, "HYDICE system: implementation and performance", Conference on Imaging Spectrometry, Orlando, FL, SPIE Conference Proceedings 2480, April 1995. 